\title{
Geometrization of metric boundary data for Einstein's equations
}

\author{
Jeffrey Winicour ${ }^{1,2}$ \\ 1 Department of Physics and Astronomy \\ University of Pittsburgh, Pittsburgh, PA 15260, USA \\ 2 Max-Planck-Institut für Gravitationsphysik, \\ Albert-Einstein-Institut, \\ 14476 Golm, Germany
}

\begin{abstract}
The principle part of Einstein equations in the harmonic gauge consists of a constrained system of 10 curved space wave equations for the components of the space-time metric. A well-posed initial boundary value problem based upon a new formulation of constraint-preserving boundary conditions of the Sommerfeld type has recently been established for such systems. In this paper these boundary conditions are recast in a geometric form. This serves as a first step toward their application to other metric formulations of Einstein's equations.
\end{abstract}

PACS numbers: PACS number(s): 04.20Ex, 04.25Dm, 04.25Nx, 04.70Bw

It is extremely satisfying to contribute this article in acknowledgment of the important influence that Jürgen Ehlers has had on my career. When I was a first year graduate student at Syracuse University in the Fall of 1959, my PhD adviser Peter Bergmann had collected an astonishing percentage of the young talent engaged in general relativity. Besides the tenured faculty members Art Komar and Richard Arnowitt, the postdocs and visiting scientists included Roy Kerr, Wolfgang Kundt, Ted Newman, Roger Penrose, Asher Peres, Ivor Robinson, Englebert Schucking, Andrej Trautman and Manfried Trumper, as well as Jürgen. At the end of that first year, when Bergmann called me to his office to discuss thesis research topics, from this group of experts it was Jürgen whom he invited to join in offering me advice. In response to Bergmann's opening question "So, what would you like to work on?", I suggested singularity structure. I had recently enjoyed reading the nice paper by Bergmann and his former student Ray Sachs on singularities in linearized gravitational theory, as well as the Einstein-Infeld-Hoffman paper on the motion of singularities. Jürgen immediately took over the conversation, "What is your underlying motivation?" I naively offered the possibility that singularity structure might be used to understand elementary particles. I then received a wide ranging lecture from Jürgen who carefully explained the conceptual problems underlying singularities even in electromagnetic theory and why the situation was much more complicated in general relativity. At that time, a global picture of the structure of the Schwarzschild singularity, which could not be anticipated from linearized theory, had just emerged with the help of Kruskal's extension of the exterior spacetime. The geometric approach of classifying singularities in terms of the incompleteness of the spacetime manifold had just begun. The important distinction between spacelike, timelike or null singularities was not yet recognized. In the following years, an effective approach to this difficult subject slowly developed from a great deal of effort by many people. Fortunately for me, I accepted Jürgen's impromptu lecture as good advice to steer clear of the subject in my graduate research.

Also at that time, general relativity was a small field going through a renaissance, which was centered in the United States about Bergmann at Syracuse and John Wheeler at Princeton. Bergmann's research, which was focused on the quantization of gravity, had led to several reformulations of Einstein's theory in terms of Lagrangian, phase space and Hamilton-Jacobi methods. But in all of these formalisms the same problems associated with dealing with nonlinearity, identifying the proper observables and handling the constraints posed the same stumbling blocks against a real physical understanding of quantum gravity that persist today. However, there were several other current developments which would have seminal impact on the future of the field: the Kruskal extension of Schwarzschild spacetime, the Kerr solution, the characteristic description of gravitational radiation by Bondi and Sachs, followed by Penrose's conformal version; Penrose's spinor description of gravity, which later led to twistor theory; and Jürgen's geometric reformulation of general relativistic hydrodynamics and thermodynamics, which set the standard for the ensuing transition in general relativity from the ambiguities of a coordinate dependent, calculational approach to a geometric approach. The discovery of quasars in the following years would bring these developments in general relativity to the attention of astrophysicists, who would become a captive audience to lectures on the geometry of curved spacetimes, thanks to Jürgen's help in organizing the first "Texas Meeting".

Throughout my career I have benefited much from Jürgen's advice, especially during my two sabbaticals with his group in Garching and my annual visits to the Albert Einstein Institute, an institute which was created due to his efforts as founding director. Through my exposure to his standards of clarity and rigor and his emphasis on a geometric picture, Jürgen endures as my mentor. I know that he would much prefer to be the cause of an article of substance rather than praise, so in that spirit I present the following discussion of the geometrical aspects of the initial-boundary value problem (IBVP) in general relativity. Quite some time ago, Jürgen and his student Saskia Kind [1] treated the spherically symmetric IBVP for a general relativistic fluid, and they later applied the work to 
stellar oscillations in collaboration with Bernd Schmidt [2]. At that time, little was known about the treatment of boundaries in general relativity outside of the spherically symmetric case. Here I treat the vacuum problem in the absence of symmetries. In recent work, catalyzed by interactions at the Albert Einstein Institute, the well-posedness of the IBVP for Einstein's equations has been established using harmonic coordinates [3]. This puts the IBVP on the same analytic footing as the Cauchy problem, whose well-posedness was also established using harmonic coordinates in the classic work of Choquet-Bruhat [4]. However, the geometric formulation of the boundary conditions and boundary data for the IBVP is more complicated than for the Cauchy problem, in which the initial data can be expressed in terms of the intrinsic metric and extrinsic curvature of the initial Cauchy hypersurface. In this article I will discuss these geometrical considerations which underlie the treatment of a boundary.

In the Cauchy problem, initial data on a spacelike hypersurface $\mathcal{S}_{0}$ are extended to a solution in the domain of dependence $\mathcal{D}\left(\mathcal{S}_{0}\right)$ (which consists of those points whose past directed characteristics all intersect $\mathcal{S}_{0}$ ). In the IBVP, data on timelike boundary $\mathcal{T}$ transverse to $\mathcal{S}_{0}$ is used to further extend the solution to the domain of dependence $\mathcal{D}\left(\mathcal{S}_{0} \cup \mathcal{T}\right)$.

The IBVP for Einstein's equations has only relatively recently widespread attention, when its importance to numerical relativity was pointed out [5]. The first well-posed IBVP was achieved for a tetrad formulation of Einstein's theory using a first differential order system which included the tetrad, the connection and the curvature tensor as evolution fields [6]. Subsequently, a well-posed IBVP was formulated for the harmonic formulation of Einstein's equations as second order wave equations for the metric [3]. This extended the classic analytic treatment of Choquet-Bruhat [4] to the well-posedness of the harmonic IBVP. The initial data for the Cauchy problem has a simple description in terms of the intrinsic metric and extrinsic curvature of $\mathcal{S}_{0}$. The aim of this article is to present an interpretation of the boundary data in the IBVP in terms of the geometry of $\mathcal{T}$. This is not only important for a clearer understanding of the nature of the gravitational IBVP but also, from a practical point of view, for the application of boundary conditions in numerical relativity. The boundary conditions developed in [3] have been successfully implemented in a evolution code based upon the harmonic formulation of Einstein's equations [7]. However, much of the numerical work in general relativity is carried out using other metric formulations, e.g. the BSSN formulation [8, 9], where well-posedness of the IBVP currently remains an unresolved issue. The geometric formulation of boundary conditions for the metric presented here is a step in that direction.

I begin with a short review of the Cauchy problem in Sec. I. followed by a discussion of the new difficulties presented by the IBVP and their analytic resolution using a harmonic formulation in Sec's. II and III where the analytic form of the boundary conditions is expressed in terms of partial derivatives of the harmonic metric. In Sec's. IV and V these are recast in covariant form in terms of geometric structures intrinsic to the IBVP.

\section{THE CAUCHY PROBLEM}

The initial data for the Cauchy problem can be formulated in a purely 3-dimensional form in terms of the intrinsic metric $h_{\mu \nu}$ and extrinsic curvature $k_{\mu \nu}$ of the initial Cauchy hypersurface. Here, for notational simplicity, I will use Greek indices rather loosely to describe either 3-dimensional or 4-dimensional objects. A major notational complication of the IBVP arises from the $3+1$ decomposition intrinsic to the Cauchy hypersurfaces and the separate $3+1$ decomposition intrinsic to the timelike boundary $\mathcal{T}$. As consistently as possible, I will use lower case letters, e.g. $h_{\mu \nu}$ and $k_{\mu \nu}$, for geometric objects associated with the Cauchy hypersurfaces and upper case letters for their counterpart on the boundary. The Cauchy data are subject to the Hamiltonian and momentum constraints

$$
0={ }^{(3)} R+\left(k_{\mu}^{\mu}\right)^{2}-k_{\mu \nu} k^{\mu \nu} \quad\left(=2 G_{\mu \nu} n^{\mu} n^{\nu}\right)
$$

and

$$
0={ }^{(3)} \nabla_{\mu}\left(k_{\nu}^{\mu}-\delta_{\nu}^{\mu} k_{\rho}^{\rho}\right) \quad\left(=2 h_{\nu}^{\mu} G_{\mu \rho} n^{\rho}\right)
$$

where ${ }^{(3)} \nabla_{\mu}$ is the covariant derivative and ${ }^{(3)} R$ is the curvature scalar associated with $h_{\mu \nu}$. Subject to these constraints, the Cauchy data determine a solution of Einstein's equations which is unique up to a diffeomorphism (cf. [10] for an exposition with many techniques common to the approach adopted here for the IBVP.)

However, as already hinted by the parenthetical appearance of the Einstein tensor $G_{\mu \nu}$ in (1.1) and (1.2), this disembodied 3-dimensional form of the Cauchy data hides the complexity of the underlying 4-dimensional space-time problem. In order to evolve the data it is necessary to introduce a foliation of the spacetime by Cauchy hypersurfaces $\mathcal{S}_{t}$, with unit timelike normal $n_{\mu}$. The evolution of the spacetime metric $g_{\mu \nu}$ is carried out along the flow of a timelike vector field $t^{\mu}$ related to the normal by the lapse $\alpha$ and shift $\beta^{\mu}$ according to

$$
t^{\mu}=\alpha n^{\mu}+\beta^{\mu}, \quad \beta^{\mu} n_{\mu}=0 .
$$


In numerical applications, the evolution is coordinatized by a time function $t$ satisfying $\mathcal{L}_{t} t=1$ and $n_{\mu}=-\alpha \nabla_{\mu} t$, and spatial coordinates satisfying $\mathcal{L}_{t} x^{i}=0$, where $\mathcal{L}_{t}$ is the Lie derivative with respect to $t^{\mu}$. In this 4 -dimensional setting, $h_{\mu \nu} n^{\nu}=0$ and the spacetime metric is given by

$$
g_{\mu \nu}=-n_{\mu} n_{\nu}+h_{\mu \nu} .
$$

An additional complexity, already apparent from the arbitrariness of the diffeomorphism freedom in the solution, is that Einstein equations do not directly provide a hyperbolic system of evolution equations. For that purpose, it is necessary to restrict the gauge freedom. This can be accomplished by introducing harmonic coordinates, i.e. four solutions $x^{\mu}=\left(t, x^{i}\right)$ of the curved space scalar wave equation $\square_{g} x^{\mu}=0$, so that in these coordinates the metric satisfies

$$
\mathcal{C}^{\mu}:=\Gamma^{\mu}=g^{\rho \sigma} \Gamma_{\rho \sigma}^{\mu}=-\frac{1}{\sqrt{-g}} \partial_{\nu} \gamma^{\nu \mu}=0,
$$

where $\gamma^{\mu \nu}=\sqrt{-g} g^{\mu \nu}$.

This leads to the standard harmonic reduction of the Einstein tensor

$$
E^{\mu \nu}:=G^{\mu \nu}-\nabla^{(\mu} \Gamma^{\nu)}+\frac{1}{2} g^{\mu \nu} \nabla_{\rho} \Gamma^{\rho},
$$

whereby the harmonic conditions (1.3) together with the vacuum Einstein equations give rise to the quasilinear system of coupled wave equations

$$
0=2 \sqrt{-g} E^{\mu \nu}=g^{\rho \sigma} \partial_{\rho} \partial_{\sigma} \gamma^{\mu \nu}+N_{\mu \nu},
$$

where $N_{\mu \nu}$ represents terms which do not enter the principle part.

In the harmonic formulation, the Hamiltonian constraints are satisfied as a consequence of the harmonic constraints (1.3) provided the initial data satisfies $\left.\Gamma^{\mu}\right|_{t=0}=0$ and $\left.\partial_{t} \Gamma^{\mu}\right|_{t=0}=0$. The harmonic conditions also determine $\partial_{t} \alpha$ and $\partial_{t} \beta$, so that the remaining freedom in the initial gauge data reduce to $\left.\alpha\right|_{t=0}$ and $\left.\beta^{\mu}\right|_{t=0}$ We return to the issue of constraint preservation in Sec. VII.

In summary, the Cauchy data necessary for determination of a unique space-time metric consist of $\left.g_{\mu \nu}\right|_{t=0}=0$ and $\left.\partial_{t} g_{\mu \nu}\right|_{t=0}=0$, subject to constraints. This 4-dimensional space-time version of the initial data is referred to as the thin sandwich formulation, as opposed to the disembodied 3-dimensional version in which $\left.h_{\mu \nu}\right|_{t=0}=\left.0 k_{\mu \nu}\right|_{t=0}=0$ are prescribed. The resulting harmonic evolution is not only unique but the solution depends continuously on the choice of initial data, i.e. the harmonic Cauchy problem is well-posed [4].

\section{DIFFICULTIES OF THE BOUNDARY TREATMENT}

The IBVP has quite different features than the Cauchy problem, as can be inferred from the simple properties of the flat-space scalar wave equation

$$
\left(\partial_{t}^{2}-\delta^{i j} \partial_{i} \partial_{j}\right) \Phi=0 \quad x^{i}=(x, y, z)
$$

in the region $x \leq 0$. For a given propagation direction $k^{i}$, there will be two characteristics (light rays) $x^{i}= \pm k^{i} t$ crossing the Cauchy hypersurface at $t=0$, but only one characteristic with $k^{x}>0$ crossing the boundary at $x=0$. As a result, although the initial Cauchy data consist of $\left.\Phi\right|_{t=0}$ and $\left.\partial_{t} \Phi\right|_{t=0}$, only half as much boundary data can be freely prescribed at $x=0$, e.g the Dirichlet data $\left.\partial_{t} \Phi\right|_{x=0}$, or the Neumann data $\left.\partial_{x} \Phi\right|_{x=0}$ or the Sommerfeld data $\left.\left(\partial_{t}+\partial_{x}\right) \Phi\right|_{x=0}$ (based upon the derivative in the outgoing characteristic direction). In the gravitational case, this inability to prescribe both the metric and its normal derivative on a timelike boundary implies that you cannot freely prescribe both the intrinsic metric of the boundary and its extrinsic curvature. In terms of the metric components, the most you can describe is a single quantity, e.g. the Sommerfeld data $K^{\mu} \partial_{\mu} g_{\rho \sigma}$ where $K^{\mu}$ is an outgoing null direction. Such a Sommerfeld boundary condition is most beneficial for numerical work since it allows discretization error to propagate across the boundary (whereas Dirichlet and Neumann boundary conditions reflect the error and trap it in the grid).

Inability to prescribe both the metric and its normal derivative, complicates constraint enforcement on the boundary, i.e. the Hamiltonian and momentum constraints cannot be enforced directly because they couple the metric and its normal derivative. Instead, the approach in [3] is to enforce the harmonic constraints $\mathcal{C}^{\mu}=0$ on the boundary and then show that this (indirectly) leads to the satisfaction of the Hamiltonian and momentum constraints. 
An additional complication is that the domain of dependence of the boundary $\mathcal{D}(\mathcal{T})$ by itself is empty. Crudely speaking, half of the past directed characteristics from each interior point do not meet the boundary. The Cauchy problem is intrinsically coupled with the boundary problem. At the intersection of $\mathcal{S}_{0}$ and $\mathcal{T}$ the Cauchy data and boundary data must be prescribed in a consistent way, or otherwise an artificial shock wave will be generated. In practice, this is hard to implement without, for example, using an exact solution in the neighborhood of the intersection. Moreover, the boundary in general moves relative to the Cauchy hypersurfaces, i.e. the normal $n_{\mu}$ to the Cauchy hypersurfaces is not in general tangent to $\mathcal{T}$. This complicates the geometric relation between the separate $3+1$ decompositions associated with the boundary and the Cauchy foliation.

Furthermore, the boundary does not pick out a unique outgoing null direction at a given point (but, instead, essentially a half null cone). This complicates the geometric formulation and interpretation of Sommerfeld boundary data. This is in addition to the issue of a physical interpretation of the boundary data. Unless the boundary is defined by a compact matter distribution, its very existence is physically artificial. This is the situation in numerical relativity where a finite outer boundary is typically introduced even though the most important numerical output might be the extraction of the gravitational waves that propagate to infinity. The treatment of such an artificial boundary can introduce spurious physical effects on the extracted waveform, similar to the effects arising from initial Cauchy data which contains spurious gravitational waves. Here I concentrate on the geometrical aspects of the boundary treatment but the underlying methods can also be used to improve the physical properties of the treatment of an isolated gravitating system [11], e.g. by the construction of boundary conditions which lead to asymptotically vanishing reflection coefficients from a sufficiently round boundary for increasingly large radius.

\section{STRONGLY WELL-POSED CONSTRAINT-PRESERVING IBVP WITH SOMMERFELD BOUNDARY CONDITIONS}

I begin the discussion of the geometrization of the boundary data with the analytic formulation of a strongly wellposed treatment of the IBVP for the Einstein equations in the harmonic gauge based upon [3, 12]. The harmonic coordinates $x^{\alpha}=\left(t, x^{i}\right)$ induce a foliation of the boundary by $t=c o n s t$ surfaces $\mathcal{B}_{t}$. This foliation determines a unique future-timelike vector unit $T^{\mu}$ which is orthogonal to $\mathcal{B}_{t}$ and tangent to the boundary. Here the metric has the status of an unknown to be determined by solving (1.5), so that other metric related quantities, such as the norm of $T^{\mu}$, have similar status. Along with the unit spacelike normal $N^{\mu}$ which points outward from the boundary, this leads to an orthonormal tetrad $\left(T^{\mu}, N^{\mu}, Q^{\mu} \cdot \bar{Q}^{\mu}\right)$ at each point on the boundary, where $Q^{\mu}$ is complex null vector tangent to $\mathcal{B}_{t}$ with normalization

$$
Q^{\mu} \bar{Q}_{\mu}=2, \quad Q^{\mu} Q_{\mu}=0 .
$$

The tetrad is uniquely determined up to the spin-rotation freedom $Q^{\mu} \rightarrow e^{i \theta} Q^{\mu}$. Uniquely associated with this tetrad (independent of the choice of $Q^{\mu}$ ) are the outgoing and ingoing null vector fields $K^{\mu}=T^{\mu}+N^{\mu}$ and $L^{\mu}=T^{\mu}-N^{\mu}$, respectively.

The outgoing null vector $K^{\mu}$ allows us to pose Sommerfeld boundary conditions. In [3] it was shown that a properly constructed hierarchy of Sommerfeld boundary conditions of the type

$$
K^{\mu} \partial_{\mu} \gamma^{\rho \sigma}=\text { Sommerfeld data, } \quad \gamma^{\mu \nu}=\sqrt{-g} g^{\mu \nu}
$$

leads to a strongly well-posed IBVP. In addition to the continuous dependence of the solution on the initial Cauchy data, strong well-posedness implies boundary stability, i.e. that the solution for the metric and its derivatives can be estimated in terms of the boundary data [13].

Certain components of the Sommerfeld data are unconstrained. These unconstrained data are picked out by the projection tensor [7]

$$
P_{\mu}^{\nu}=\delta_{\mu}^{\nu}+\frac{1}{2} L_{\mu} K^{\nu}
$$

which projects a 1 -form $V_{\mu}$ into the $\left(K_{\mu}, Q_{\mu}, \bar{Q}_{\mu}\right)$ subspace.

The freely prescribed Sommerfeld data $q^{\rho \sigma}$ are the 6 components

$$
P_{\alpha}^{\rho} P_{\beta}^{\sigma} K^{\mu} \partial_{\mu} \gamma^{\alpha \beta}=q^{\rho \sigma},
$$

where $q^{\rho \sigma} L_{\sigma}=0$. The remaining boundary conditions enforce the harmonic constraints $\left.\mathcal{C}^{\nu}\right|_{\mathcal{T}}=0$ by expressing them in the form

$$
\sqrt{-g} \mathcal{C}^{\nu}=\sqrt{-g} \Gamma^{\nu}=-\partial_{\rho} \gamma^{\rho \nu}=+\frac{1}{2} L_{\rho} K^{\mu} \partial_{\mu} \gamma^{\rho \nu}-P_{\rho}^{\mu} \partial_{\mu} \gamma^{\rho \nu}-P_{\alpha}^{\rho} P_{\beta}^{\sigma} K^{\mu} \partial_{\mu} \gamma^{\alpha \beta}=0
$$


which provide Sommerfeld conditions for the remaining $L_{\rho}$ components of $K^{\mu} \partial_{\mu} \gamma^{\rho \nu}$.

Strong well-posedness results from the hierarchical structure of the boundary conditions (3.3) and (3.4), i.e. the boundary conditions must form a sequence whose Sommerfeld data only depends on prior members. An example, which plays an important role in Sec. IV] is to prescribe the unconstrained components (3.3) in the sequence

$$
\begin{aligned}
\frac{1}{2} K_{\rho} K_{\sigma} K^{\mu} \partial_{\mu} \gamma^{\rho \sigma} & =-\sqrt{-g} q_{K K}, \\
\left(Q_{\rho} K_{\sigma} K^{\mu}-\frac{1}{2} K_{\rho} K_{\sigma} Q^{\mu}\right) \partial_{\mu} \gamma^{\rho \sigma} & =-\sqrt{-g} q_{K Q}, \\
\left(Q_{\rho} \bar{Q}_{\sigma} K^{\mu}-\frac{1}{2} K_{\rho} K_{\sigma} L^{\mu}\right) \partial_{\mu} \gamma^{\rho \sigma} & =-\sqrt{-g} q_{Q \bar{Q}} \\
\left(\frac{1}{2} Q_{\rho} Q_{\sigma} K^{\mu}-Q_{\rho} K_{\sigma} Q^{\mu}\right) \partial_{\mu} \gamma^{\rho \sigma} & =-\sqrt{-g} q_{Q Q}
\end{aligned}
$$

and then to add on the constrained components (3.4) in the sequence

$$
\begin{aligned}
& 2 \sqrt{-g} K_{\mu} \Gamma^{\mu}=\left(K_{\rho} L_{\sigma} K^{\mu}+K_{\rho} K_{\sigma} L^{\mu}-K_{\rho} \bar{Q}_{\sigma} Q^{\mu}-K_{\rho} Q_{\sigma} \bar{Q}^{\mu}\right) \partial_{\mu} \gamma^{\rho \sigma}=0, \\
& 2 \sqrt{-g} Q_{\mu} \Gamma^{\mu}=\left(L_{\rho} Q_{\sigma} K^{\mu}+K_{\rho} Q_{\sigma} L^{\mu}-Q_{\rho} \bar{Q}_{\sigma} Q^{\mu}+Q_{\rho} Q_{\sigma} \bar{Q}^{\mu}\right) \partial_{\mu} \gamma^{\rho \sigma}=0, \\
& 2 \sqrt{-g} L_{\mu} \Gamma^{\mu}=\left(L_{\rho} L_{\sigma} K^{\mu}+K_{\rho} L_{\sigma} L^{\mu}-\bar{Q}_{\rho} L_{\sigma} Q^{\mu}-Q_{\rho} L_{\sigma} \bar{Q}^{\mu}\right) \partial_{\mu} \gamma^{\rho \sigma}=0 .
\end{aligned}
$$

The sequential structure is determined by the order

$$
\left(K_{\rho} K_{\sigma}\right),\left(K_{\rho} Q_{\sigma}\right),\left(Q_{\rho} \bar{Q}_{\sigma}\right),\left(Q_{\rho} Q_{\sigma}\right),\left(K_{\rho} L_{\sigma}\right),\left(Q_{\rho} L_{\sigma}\right),\left(L_{\rho} L_{\sigma}\right)
$$

in which the components of $\partial_{\mu} \gamma^{\rho \sigma}$ enter into the Sommerfeld boundary condition for $K^{\mu} \partial_{\mu} \gamma^{\rho \sigma}$.

The boundary conditions (3.5) - (3.8) can be modified by the addition of lower differential order terms without affecting strong well-posedness. Such terms can be used to reduce spurious back reflections from the boundary of an isolated system. (See [11] for more details.) In addition, the boundary conditions (3.9) - (3.11) can also be modified in the form

$$
\Gamma^{\mu}-H^{\mu}(x . g)=0
$$

to include gauge forcing terms $H^{\mu}$ corresponding to a generalized harmonic formulation. Other examples of Sommerfeld boundary conditions that fit into the hierarchical structure and that ensure strong well-posedness are considered in 7, 11]

The physical content of these boundary conditions can be clarified by considering the case of a linearized wave incident on a plane boundary. The first three pieces of free boundary data $\left(q_{K K}, q_{K Q}, q_{Q \bar{Q}}\right)$ are related to the gauge freedom, i.e. they can be set to zero without loss of generality. The next piece of data $\left(q_{Q Q}\right)$ controls the gravitational radiation. The remaining conditions enforce the constraints $\mathcal{C}^{\mu}=0$ on the boundary. This is consistent with the physical expectation that two pieces (or one complex piece) of data are necessary to describe the two polarization degrees of freedom of a gravitational wave. More generally, the boundary has curvature, which necessitates additional nonzero boundary data, as discussed out in [11]. In general, the curvature of the boundary combines with the radiation modes in a way which cannot be cleanly separated. This is one of the complications emphasized in the treatment given in [6]. It is an issue I return to in Sec. VIB I next consider how to reverse engineer the above Sommerfeld boundary conditions to clarify their geometrical content.

\section{BOUNDARY GEOMETRY}

I proceed using 4-dimensional notation to describe the 3-dimensional geometrical objects intrinsic to the boundary. At the most primitive level, these are the intrinsic 3-metric

$$
H_{\mu \nu}=g_{\mu \nu}-N_{\mu} N_{\nu}
$$

its corresponding 3-connection $D_{\mu}$ and the extrinsic curvature

$$
\mathcal{K}_{\mu \nu}=H_{\mu}^{\rho} \nabla_{\rho} N_{\nu}
$$

However, those objects are insufficient to formulate Sommerfeld boundary conditions because they do not determine a unique outward null direction. 
For that purpose, we introduce an evolution gauge vector field $t^{\mu}$ tangent to the boundary. This is in analogy with what is done in a numerical evolution the Cauchy problem but for the moment we need only define $t^{\mu}$ on the boundary and not in the interior. It is not necessary that $t^{\mu}$ be timelike but its flow must map, in a future-directed sense, the edge $\mathcal{B}_{0}$ (where the boundary intersects the initial Cauchy hypersurface) into a smooth foliation $\mathcal{B}_{t}$ of the boundary. The foliation is parameterized by a time function $t$ satisfying $\mathcal{L}_{t} t=1$, with $t=0$ on $\mathcal{B}_{0}$. (Here $\mathcal{L}_{t}$ represents the Lie derivative with respect to $t^{\mu}$.) In turn, along with the intrinsic metric of the boundary, the 2 -surfaces $\mathcal{B}_{t}$ determine a unit timelike normal field tangent to the boundary according to $T_{\mu}=-A D_{\mu} t=-A H_{\mu}^{\nu} \nabla_{\mu} t$. The corresponding lapse $A$ and shift $B^{\mu}$ intrinsic to the boundary are defined according to $t^{\mu}=A T^{\mu}+B^{\mu}$.

The intrinsic 2-metric of the boundary foliation is given by

$$
Q_{\mu \nu}=Q_{(\mu} \bar{Q}_{\nu)}=H_{\mu \nu}-T_{\mu} T_{\nu}
$$

where we have again introduced a complex null vector $Q^{\mu}$ with the normalization (3.1). The extrinsic curvature of this foliation associated with the normal $T^{\mu}$ is

$$
\kappa_{\mu \nu}=Q_{\mu}^{\rho} D_{\rho} T_{\nu}
$$

This structure is sufficient to geometrize the last piece of free Sommerfeld data $q_{Q Q}$ in the hierarchy (3.5) - (3.8) by relating it to the extrinsic curvature tensors of $\mathcal{B}_{t}$ according to

$$
Q^{\rho} Q^{\sigma}\left(\mathcal{K}_{\rho \sigma}+\kappa_{\rho \sigma}\right)=\frac{1}{2} Q^{\rho} Q^{\sigma} K^{\mu} \partial_{\mu} g_{\rho \sigma}-Q^{\rho} K^{\sigma} Q^{\mu} \partial_{\mu} g_{\rho \sigma}=q_{Q Q}
$$

Equivalently, we can express this data in terms of the optical shear $\sigma$ of the outgoing null congruence determined by $K^{\mu}$

$$
2 \sigma=Q^{\rho} Q^{\sigma} \nabla_{\rho} K_{\sigma}=q_{Q Q} .
$$

In linearized theory, $\sigma$ is the complex data for an ingoing gravitational wave incident on a plane or spherical boundary.

The free data for the first 3 Sommerfeld conditions (3.5) - (3.7) cannot be similarly expressed in terms of the geometry of the boundary and its tangent space. For example, the first condition, rewritten in terms of the metric, is

$$
\frac{1}{2} K^{\rho} K^{\sigma} K^{\mu} \partial_{\mu} g_{\rho \sigma}=q_{K K}
$$

Since $K^{\mu}=T^{\mu}+N^{\mu}$, this contains pieces such as $N^{\rho} N^{\sigma} N^{\mu} \partial_{\mu} g_{\rho \sigma}$ which can be set to zero by a diffeomorphism leaving the boundary and its extrinsic curvature intact, e.g. by introducing Gaussian normal coordinates adapted to the boundary. A geometric formulation of these boundary conditions requires additional structure to eliminate the effect of this gauge freedom. One convenient approach is to introduce a preferred background geometry, as described next.

\section{INTRODUCTION OF A BACKGROUND METRIC ASSOCIATED WITH THE INITIAL DATA}

Geometrization of the boundary conditions (3.5) - (3.7) can be accomplished by introducing a background metric $\stackrel{\circ}{g}_{\mu \nu}$ with its associated Christoffel symbols $\stackrel{\circ}{\Gamma}_{\mu \nu}^{\rho}$ and curvature tensor $\stackrel{\circ}{R}_{\rho \sigma(\mu}^{\lambda}$. Setting $f_{\mu \nu}=g_{\mu \nu}-\stackrel{\circ}{g}_{\mu \nu}$, the gauge freedom in first derivatives of the metric is then fixed relative to the background by the tensor field

$$
C_{\mu \nu}^{\rho}:=\Gamma_{\mu \nu}^{\rho}-\stackrel{\circ}{\Gamma}_{\mu \nu}^{\rho}=\frac{1}{2} g^{\rho \sigma}\left(\stackrel{\circ}{\nabla}_{\mu} f_{\nu \sigma}+\stackrel{\circ}{\nabla}_{\nu} f_{\mu \sigma}-\stackrel{\circ}{\nabla}_{\sigma} f_{\mu \nu}\right) .
$$

(In order to avoid confusion, raising and lowering of indices will only be done with the physical metric $g_{\mu \nu}$ unless otherwise noted.)

The harmonic constraints are now modified to the covariant form

$$
\mathcal{C}^{\rho}:=g^{\mu \nu}\left(\Gamma_{\mu \nu}^{\rho}-\stackrel{\circ}{\Gamma_{\mu \nu}^{\rho}}\right)=0,
$$

or

$$
\mathcal{C}^{\rho}:=g^{\mu \nu}\left(\Gamma_{\mu \nu}^{\rho}-\stackrel{\circ}{\Gamma}_{\mu \nu}^{\rho}\right)-H^{\rho}=0
$$


if a gauge forcing term $H^{\rho}(x, g)$ is included.

Einstein's equations for $f_{\mu \nu}$ reduce to the quasilinear wave system

$$
g^{\rho \sigma} \nabla_{\rho}^{\circ} \stackrel{\circ}{\nabla}_{\sigma} f_{\mu \nu}=2 g_{\lambda \tau} g^{\rho \sigma} C_{\mu \rho}^{\lambda} C_{\nu \sigma}^{\tau}+4 C_{\sigma(\mu}^{\rho} g_{\nu) \lambda} C_{\rho \tau}^{\lambda} g^{\sigma \tau}-2 g^{\rho \sigma} \stackrel{\circ}{R}_{\rho \sigma(\mu}^{\lambda} g_{\nu) \lambda}+2 \nabla_{(\mu} H_{\nu)}
$$

for which the formulation of a well-posed IBVP goes through exactly as before. (See 11] for details.) The null tetrad $\left(K^{\mu}, L^{\mu}, Q^{\mu}, \bar{Q}^{\mu}\right)$ associated with the physical metric $g_{\mu \nu}$ is again defined on the boundary in terms of the foliation constructed by introducing an evolution field $t^{\mu}$ tangent to the boundary. This null tetrad is then used to prescribe a hierarchical set of Sommerfeld boundary conditions. In terms of $f_{\mu \nu}$ the analogue of (3.5) - (3.11) take the hierarchical form

$$
\begin{aligned}
& \frac{1}{2} K^{\rho} K^{\sigma} K^{\mu} \stackrel{\circ}{\mu}_{\mu} f_{\rho \sigma}=q_{K} \\
& \left(Q^{\rho} K^{\sigma} K^{\mu} \stackrel{\circ}{\nabla}_{\mu}-\frac{1}{2} K^{\rho} K^{\sigma} Q^{\mu} \stackrel{\circ}{\nabla}_{\mu}\right) f_{\rho \sigma}=q_{Q}, \\
& \left(L^{\rho} K^{\sigma} K^{\mu} \stackrel{\circ}{\nabla}_{\mu}-\frac{1}{2} K^{\rho} K^{\sigma} L^{\mu} \stackrel{\circ}{\nabla}_{\mu}\right) f_{\rho \sigma}=q_{L}, \\
& \left(\frac{1}{2} Q^{\rho} Q^{\sigma} K^{\mu} \stackrel{\circ}{\mu}_{\mu}-Q^{\rho} K^{\sigma} Q^{\mu} \nabla_{\mu}\right) f_{\rho \sigma}=q_{Q Q}, \\
& \left(Q^{\rho} \bar{Q}^{\sigma} K^{\mu}+K^{\rho} K^{\sigma} L^{\mu}-K^{\rho} \bar{Q}^{\sigma} Q^{\mu}-K^{\rho} Q^{\sigma} \bar{Q}^{\mu}\right) \stackrel{\circ}{\nabla}_{\mu} f_{\rho \sigma}=-2 K^{\mu} H_{\mu}, \\
& \left(L^{\rho} Q^{\sigma} K^{\mu}+K^{\rho} Q^{\sigma} L^{\mu}-K^{\rho} L^{\sigma} Q^{\mu}+Q^{\rho} Q^{\sigma} \bar{Q}^{\mu}\right) \stackrel{\circ}{\nabla}_{\mu} f_{\rho \sigma}=-2 Q^{\mu} H_{\mu}, \\
& \left(L^{\rho} L^{\sigma} K^{\mu}+Q^{\rho} \bar{Q}^{\sigma} L^{\mu}-\bar{Q}^{\rho} L^{\sigma} Q^{\mu}-Q^{\rho} L^{\sigma} \bar{Q}^{\mu}\right) \stackrel{\circ}{\nabla}_{\mu} f_{\rho \sigma}=-2 L^{\mu} H_{\mu} .
\end{aligned}
$$

Note that these conditions can be modified by taking linear combinations consistent with the hierarchy. For example, by subtracting (5.2) we can replace (5.4) by

$$
\left(N^{\rho} K^{\sigma} K^{\mu} \stackrel{\circ}{\mu}_{\mu}-\frac{1}{2} K^{\rho} K^{\sigma} N^{\mu} \nabla_{\mu}\right) f_{\rho \sigma}=q_{N} .
$$

The Sommerfeld boundary conditions (5.2) - (5.8) for $f_{\rho \sigma}$ are now in covariant form and the geometrization the boundary conditions reduces to eliminating the ambiguity in the choice of the background metric. We do this by extending the evolution vector field $t^{\mu}$ to a neighborhood of the boundary. This fixes an "evolution gauge" which allows us to Lie transport the initial Cauchy data into the future.

The simplest choice is of background metric is to set

$$
\left.\stackrel{\circ}{g}_{\mu \nu}\right|_{t=0}=\left.g_{\mu \nu}\right|_{t=0}, \quad \mathcal{L}_{t} \stackrel{\circ}{\mu \nu}_{\mu \nu}=0 .
$$

However, higher order compatibility with the initial Cauchy data can be incorporated by setting

$$
\left.\stackrel{\circ}{g}_{\mu \nu}\right|_{t=0}=\left.g_{\mu \nu}\right|_{t=0}, \quad \mathcal{L}_{t} \stackrel{\circ}{\mu \nu}_{\mu \nu}=\left.\mathcal{L}_{t} g_{\mu \nu}\right|_{t=0} .
$$

A further option of (5.11) is to to require that the the background metric is harmonic. This is most readily expressed in the coordinates $x^{\mu}=\left(t, x^{i}\right)$ adopted to the evolution according to

$$
\mathcal{L}_{t} t=1, \quad \mathcal{L}_{t} x^{i}=0
$$

Then the densitized background metric

$$
\sqrt{-\stackrel{g}{g} g^{\mu \nu}}=a^{\mu \nu}+b^{\mu \nu} t+\frac{1}{2} c^{\mu \nu} t^{2}+\frac{1}{6} d^{\mu \nu} t^{3},
$$

where $a^{\mu \nu}$ and $b^{i j}$ are determined by the initial Cauchy data, satisfies the harmonicity condition provided

$$
\begin{aligned}
c^{i j} & =0, \quad d^{\mu i}=0, \\
b^{t t} & =-\partial_{i} a^{t i}, \quad b^{t i}=-\partial_{j} a^{i j}, \\
c^{t t} & =-\partial_{i} b^{t i}=\partial_{i} \partial_{j} a^{i j}, \\
c^{t i} & =-\partial_{j} b^{i j} d^{t t}=-\partial_{i} c^{t i}=\partial_{i} \partial_{j} b^{i j} .
\end{aligned}
$$

(Here $g^{\mu \nu}$, the inverse of $\stackrel{\circ}{g}_{\mu \nu}$, is used to raise indices.) Other background metrics associated with Lie transport of the initial Cauchy data could also be used. 
For both the alternatives (5.11) and (5.13), $f_{\mu \nu}=g_{\mu \nu}-\stackrel{\circ}{g}_{\mu \nu}$ has homogeneous (vanishing) initial Cauchy data so that they provide the first approximation to a standard iterative scheme to prove existence of solutions of the quasilinear problem [13]. From a practical point of view, they also automatically ensure a $C^{1}$ compatibility between the initial data and boundary data, and thus in a numerical evolution would reduce spurious high frequency waves arsing at the edge $\mathcal{B}_{0}$. Such a homogeneous version of the initial value problem can also be useful in numerical simulations by eliminating the effect of extreme nonlinear behavior.

In all three cases, (5.10), (5.11) and (5.13), the background $\stackrel{\circ}{g}_{\mu \nu}$ is uniquely defined by the choice of the evolution vector $t^{\mu}$. This applies to any metric based formulation of the Cauchy problem for Einstein's equations. Note that all such choices of $t^{\mu}$ are related by diffeomorphism, so although $t^{\mu}$ is a critical ingredient it is a pure gauge object. It does not determine metric information without further knowledge of the lapse and shift.

Given a choice of evolution vector $t^{\mu}$, the diffeomorphism freedom in the solution to the harmonic IBVP can be completely eliminated by tying it to the adapted coordinates determined by a specific coordinatization of $\mathcal{S}_{0}$ according to (5.12). Then the results of [11] guarantee the local existence of a unique solution to the generalized harmonic equations (5.1) subject to the boundary conditions (5.2) - (5.8).

\section{GEOMETRICAL INTERPRETATION OF THE SOMMERFELD DATA}

A geometrical interpretation of the Sommerfeld boundary data in (5.2) - 5.5) can be made by replacing the derivatives $\stackrel{\circ}{\nabla}_{\mu} f_{\rho \sigma}$ in terms of derivatives of $K_{\sigma}$. The identity $\left(\nabla_{\rho}-\stackrel{\circ}{\nabla_{\rho}}\right) K_{\sigma}=-C_{\rho \sigma}^{\mu} K_{\mu}=\frac{1}{2} K^{\mu}\left(\stackrel{\circ}{\nabla}_{\mu} f_{\rho \sigma}-\stackrel{\circ}{\nabla}_{\rho} f_{\mu \sigma}-\stackrel{\circ}{\nabla_{\sigma}} f_{\mu \rho}\right)$ can be used to rewrite (5.5) in terms of the optical shear of the boundary foliation,

$$
2(\sigma-\stackrel{\circ}{\sigma})=Q^{\rho} Q^{\sigma}\left(\nabla_{\rho}-\stackrel{\circ}{\nabla}_{\rho}\right) K_{\sigma}=\frac{1}{2} Q^{\rho} Q^{\sigma} K^{\mu} \stackrel{\circ}{\nabla}_{\mu} f_{\rho \sigma}-Q^{\rho} K^{\sigma} Q^{\mu} \stackrel{\circ}{\nabla}_{\mu} f_{\rho \sigma} \cdot=q_{Q Q}
$$

Analogous to (4.3), this equates the Sommerfeld data $q_{Q Q}$ to the shear of the outgoing null hypersurfaces determined by the boundary foliation, relative to the background value of the shear. Alternatively, the spin-weight dependence of this relative shear can be removed by expressing it as the rank two symmetric tensor

$$
\tilde{q}_{\mu \nu}:=2\left(\sigma_{\mu \nu}-\stackrel{\circ}{\sigma}_{\mu \nu}\right)=\left(Q_{\mu}^{\rho} Q_{\nu}^{\sigma}-\frac{1}{2} Q_{\mu \nu}\right)\left(\nabla_{\rho}-\stackrel{\circ}{\nabla}_{\rho}\right) K_{\sigma}
$$

The identity

$$
V_{\mu} K^{\rho}\left(\nabla_{\rho}-\stackrel{\circ}{\nabla}_{\rho}\right) K^{\mu}=\left(V^{\rho} K^{\sigma} K^{\mu}-\frac{1}{2} K^{\rho} K^{\sigma} V^{\mu}\right) \stackrel{\circ}{\nabla}_{\mu} f_{\rho \sigma}
$$

which holds for any vector field $V^{\mu}$, can be used to interpret the boundary data for the leading conditions (5.2) - (5.4) in the hierarchy. The geodesic acceleration of $K^{\mu}$ with respect to the physical metric and the background metric are $a^{\mu}=K^{\nu} \nabla_{\nu} K^{\mu}$ and $\stackrel{\circ}{a}{ }^{\mu}=K^{\nu} \nabla_{\nu}^{\circ} K^{\mu}$. Thus (5.2) - (5.4) can be re-expressed as

$$
\begin{aligned}
K_{\mu}\left(a^{\mu}-\stackrel{\circ}{a}^{\mu}\right)=\frac{1}{2} K^{\rho} K^{\sigma} K^{\mu} \dot{\circ}_{\mu} f_{\rho \sigma} & =q_{K}, \\
Q_{\mu}\left(a^{\mu}-\stackrel{\circ}{a}^{\mu}\right)=\left(Q^{\rho} K^{\sigma} K^{\mu}-\frac{1}{2} K^{\rho} K^{\sigma} Q^{\mu}\right) \stackrel{\circ}{\nabla}_{\mu} f_{\rho \sigma} & =q_{Q}, \\
L_{\mu}\left(a^{\mu}-\stackrel{\circ}{a}^{\mu}\right)=\left(L^{\rho} K^{\sigma} K^{\mu}-\frac{1}{2} K^{\rho} K^{\sigma} L^{\mu}\right) \stackrel{\circ}{\nabla}_{\mu} f_{\rho \sigma} & =q_{L},
\end{aligned}
$$

where the Sommerfeld data $\left(q_{K}, q_{Q}, q_{L}\right)$ is related to the acceleration by the components of

$$
q^{\mu}=\left(a^{\mu}-\stackrel{\circ}{a}^{\mu}\right)
$$

Note that neither $a^{\mu}$ nor $\stackrel{i}{a}^{\mu}$ are uniquely determined by our prior construction which only defines $K^{\mu}$ in the tangent space of the boundary. However, their difference is geometrically and uniquely defined, even though either $a^{\mu}$ or $a^{\mu}$ can be individually set to zero by extending $K^{\mu}$ inside the boundary as the tangent vector to the affinely parametrized null geodesic determined by either the connection $\nabla_{\mu}$ or $\stackrel{\circ}{\nabla}_{\mu}$, respectively.

Equations (6.1) - (6.5) show how the unconstrained Sommerfeld data can be described in terms of geometrical objects consisting of the shear and acceleration of the outgoing null vector $K^{\mu}$ relative to their background values. This raises the question whether another geometrical description can be given which does not introduce a background metric. For the shear $\sigma$, we already saw that this was possible by using (4.3). We can investigate the case of $a^{\mu}$ by decomposing 
it into the pieces $a^{\mu}=T^{\nu} \nabla_{\nu} K^{\mu}+N^{\nu} \nabla_{\nu} K^{\mu}$. The first piece $T^{\nu} \nabla_{\nu} K^{\mu}$ is intrinsic to the boundary geometry and can be expressed in terms of the extrinsic curvature of the boundary foliation according to the components

$$
\begin{aligned}
K_{\mu} T^{\nu} \nabla_{\nu} K^{\mu} & =0 \\
Q_{\mu} T^{\nu} \nabla_{\nu} K^{\mu} & =Q^{\mu} T^{\nu} \mathcal{K}_{\mu \nu}+Q^{\mu} \partial_{\mu} \log A, \\
L_{\mu} T^{\nu} \nabla_{\nu} K^{\mu} & =2 T^{\mu} T^{\nu} \mathcal{K}_{\mu \nu},
\end{aligned}
$$

where $A$ is the lapse intrinsic to the boundary and $\mathcal{K}_{\mu \nu}$ is its extrinsic curvature. Consequently, if we were to prescribe Dirichlet boundary conditions for the metric then the terms $N^{\nu} \nabla_{\nu} K^{\mu}$ would not enter and the data would be "boundary intrinsic". No background metric would then be necessary. However, the strong well-posedness of the IBVP has not been established for the case of Dirichlet boundary conditions. Furthermore, from a practical viewpoint, homogeneous Dirichlet boundary conditions are of the reflecting type so that, with the correct prescription of Dirichlet boundary data, a gravitational wave could propagate across the boundary but in numerical applications the error would be trapped in the grid.

It is the $N^{\nu} \nabla_{\nu} K^{\mu}$ piece of $a^{\mu}$ which requires an extension of $K^{\mu}$ inside the boundary. This leads to a more complicated geometric description, which depends upon the particular way that $K^{\mu}$ is extended. For instance, a boundary defining function $\Phi$ might be introduced such that $\left.\Phi\right|_{\mathcal{T}}=0$ and $\left.N_{\mu}\right|_{\mathcal{T}}=\left.\eta \nabla_{\mu} \Phi\right|_{\mathcal{T}}$, with $\eta=\left(g^{\mu \nu} \nabla_{\mu} \Phi \nabla_{\nu} \Phi\right)^{-1 / 2}$. This defines an extension of $N_{\mu}=\eta \nabla_{\mu} \Phi$ to a neighborhood of the boundary, where $\eta$ plays the role of the "lapse" for the boundary defining function. It then follows that

$$
N^{\nu} \nabla_{\nu} N_{\mu}=N_{\mu} N^{\nu} \nabla_{\nu} \log \eta-\nabla_{\mu} \log \eta=-D_{\mu} \log \eta .
$$

Next, by extending $T^{\mu}$ off the boundary by the parallel transport $N^{\nu} \nabla_{\nu} T^{\mu}=0$, we have $N^{\nu} \nabla_{\nu} K^{\mu}=N^{\nu} \nabla_{\nu} N^{\mu}$. Thus, by putting together the pieces (6.7) and (6.8), $a^{\mu}$ can be expressed in terms of the extrinsic curvature and the lapses $A$ and $\eta$. However, this requires introducing auxiliary quantities, i.e. $\Phi$ and the extension of the tetrad vectors $N^{\mu}$ and $T^{\mu}$ off the boundary. Consequently, it does not provide any simpler a geometrical description of the boundary data than the use of a background metric, except for frame-based formulations, such as [6], in which the tetrad vectors are evolution variables.

\section{A. Edge data}

The uniqueness of the solution to the harmonic IBVP requires the specification of the initial values of the lapse and shift, i.e. the relation $t^{\mu}=\alpha n^{\mu}+\beta^{\mu}$ between the evolution vector $t^{\mu}$ and the unit timelike normal $n^{\mu}$ to the initial Cauchy hypersurface $\mathcal{S}_{0}$. For the pure Cauchy problem this data is pure gauge information but for the IBVP it contains geometric information at the edge where $\mathcal{S}_{0}$ intersects the boundary $\mathcal{T}$. This edge data is the hyperbolic angle sinh $\Theta=n^{\mu} N_{\mu}$ formed by the intersection, which describes the intrinsic motion of the boundary relative to the Cauchy foliation. The tangency of the evolution vector $t^{\mu}$ to the boundary implies that $\sinh \Theta$ is related to the shift by

$$
\sinh \Theta=\frac{1}{\alpha} \beta^{\mu} N_{\mu}
$$

\section{B. Dynamics of the boundary}

The data for a linearized gravitational wave incident on a plane or spherical boundary in a background Minkowski space can be prescribed in terms of the shear of the outgoing null hypersurfaces. However, if the boundary has a more dynamical behavior, so that its intrinsic metric and extrinsic curvature change dynamically in time, then this description breaks down. In the generic case, such a boundary gives rise to a dynamically changing shear even in the absence of linearized curvature. Thus the shear must be coupled with data determining the dynamics of the boundary in order to unambiguously describe the full physical or geometrical content of the boundary data.

In the nonlinear theory, the boundary is dynamically traced out by the integral curves of the unit vector $T^{\mu}$. (It is also traced out by the integral curves of $t^{\mu}$ but this description is devoid of metrical content.) Given the initial value of $T^{\mu}$, which is supplied by the initial Cauchy and edge data, these integral curves can be constructed in principle from the geodesic curvature

$$
A^{\mu}=T^{\nu} \nabla_{\nu} T^{\mu} .
$$


(The qualification "in principle" is a reminder that the required spacetime metric and connection are unknowns until the IBVP is solved.) However, only the normal component of $N_{\mu} A^{\mu}$ enters into determining the dynamics of the boundary. We immediately have

$$
T_{\mu} A^{\mu}=0
$$

and, since $T_{\mu}=-A D_{\mu} t$ (where $A$ is the lapse internal to the boundary and $D_{\mu}$ its internal connection), we also have

$$
Q_{\mu} A^{\mu}=-Q^{\mu} T^{\nu} D_{\nu}\left(A D_{\mu} t\right)=-Q^{\mu} T^{\nu} A D_{\mu} D_{\nu} t=Q^{\mu} D_{\mu} \log A .
$$

Thus the components of $A^{\mu}$ tangential to the boundary describe the freedom corresponding to the lapse in the foliation of the boundary.

The essential piece of data that controls $N_{\mu} A^{\mu}$ in the boundary conditions (6.1) - (6.5) is $L_{\mu} q^{\mu}=L_{\mu}\left(a^{\mu}-a^{\mu}\right)$. A straightforward calculation gives

$$
L_{\mu} q^{\mu}=-2 N_{\mu} A^{\mu}+L_{\mu} N^{\nu}\left(\nabla_{\nu}-\stackrel{\circ}{\nabla}_{\nu}\right) K^{\mu}-L^{\mu} T^{\nu} \stackrel{\circ}{\nabla}_{\nu} K^{\mu},
$$

which is independent of the extension of the tetrad vectors off the boundary. Since the boundary conditions determine a unique solution of the IBVP, (6.10) uniquely determines the boundary values of $N_{\mu} A^{\mu}$. Hence, the dynamical properties of the boundary are controlled in the statement of the IBVP by the data $L_{\mu} q^{\mu}$, although in a very implicit manner.

\section{CONSTRAINT PRESERVATION}

The preservation of the harmonic constraints,

$$
\mathcal{C}^{\rho}:=g^{\mu \nu}\left(\Gamma_{\mu \nu}^{\rho}-\stackrel{\circ}{\Gamma^{\rho}}{ }_{\mu \nu}\right)-H^{\rho}(x, g)=0
$$

follows from applying the Bianchi identities to the reduced evolution system. Consider any formulation of Einstein's equations for which the reduced evolution equations $E^{\mu \nu}=0$ take the form

$$
E^{\mu \nu}:=G^{\mu \nu}-\nabla^{(\mu} \mathcal{C}^{\nu)}+\frac{1}{2} g^{\mu \nu} \nabla_{\rho} \mathcal{C}^{\rho}+A_{\sigma}^{\mu \nu} \mathcal{C}^{\sigma}=0,
$$

for some smooth coefficients $A_{\sigma}^{\mu \nu}(x, g, \partial g)$. (This includes constraint modified versions of the generalized harmonic system.) The Bianchi identity $\nabla_{\mu} G^{\mu \nu}=0$ then implies a homogeneous wave equation for $\mathcal{C}^{\mu}$,

$$
\nabla^{\rho} \nabla_{\rho} \mathcal{C}^{\mu}+R_{\rho}^{\mu} \mathcal{C}^{\rho}-2 \nabla_{\rho}\left(A_{\sigma}^{\mu \rho} \mathcal{C}^{\sigma}\right)=0 .
$$

If the boundary conditions enforce $\left.\mathcal{C}^{\rho}\right|_{\mathcal{T}}=0$ and the initial data enforces $\left.\mathcal{C}^{\rho}\right|_{\mathcal{S}_{0}}=\left.\partial_{t} \mathcal{C}^{\rho}\right|_{\mathcal{S}_{0}}=0$ then the unique solution of (7.2) is $\mathcal{C}^{\rho}=0$. As a result, the Sommerfeld boundary conditions in the geometrical form (6.2) - (6.5) along with (5.6) - (5.8), which enforce $\left.\mathcal{C}^{\rho}\right|_{\mathcal{T}}=0$, lead to a well-posed harmonic IBVP in which the constraints $\mathcal{C}^{\rho}=0$ are satisfied everywhere. In turn, (7.1) implies that the Hamiltonian and momentum constraints $G^{\mu \nu} n_{\nu}=0$ are also satisfied.

While these Sommerfeld boundary conditions can be formally applied to any metric formulation of the reduced Einstein equations, an independent check is necessary to determine whether the Hamiltonian and momentum constraints are preserved for formulations which do not explicitly contain an evolution system of the form (7.1). An important example is the BSSN system [8, 9] which is widely used in numerical simulations of binary black holes. In current numerical work, the boundary conditions for BSSN evolution systems are applied in a naive, homogeneous Sommerfeld form to each evolution variable (cf. [14]). Constraint preservation does not hold for harmonic evolution with these naive boundary conditions and cannot be expected to hold for other systems. The geometric nature of the boundary conditions (6.2) - (6.5) suggest that they could be applicable to the BSSN system, although the boundary conditions (5.6) - (5.8) enforcing the harmonic constraints would undoubtedly need modification. This issue deserves further investigation.

\section{SUMMARY}

Beginning with an analytic description of a strongly well-posed version of the (generalized) harmonic IBVP, we have shown how the boundary conditions on the metric may be expressed in a geometric form. The end result can 
be summarized as follows. On a manifold $\mathcal{M}$ with boundaries $\mathcal{S}_{0}$ and $\mathcal{T}$ meeting in an edge $\mathcal{B}_{0}$, we introduce an evolution vector field $t^{\mu}$ whose streamlines are tangent to the boundary and provide a smooth foliation $\mathcal{S}_{t}$ of $\mathcal{M}$ which intersects the boundary in in a smooth foliation $\mathcal{B}_{t}$. Although $t^{\mu}$ contains no metric information, it supplies the essential gauge information to (i) introduce a background metric on $\mathcal{M}$ by the Lie transport of the Cauchy data

$\left.g_{\mu \nu}\right|_{t=0}$ and $\left.\mathcal{L}_{t} g_{\mu \nu}\right|_{t=0}$ on $\mathcal{S}_{0}$ and (ii) introduce a null tetrad on $\mathcal{T}$ which is adapted to the foliation $\mathcal{B}_{t}$. Sommerfeld boundary data is then prescribed for the relative acceleration $q^{\mu}$, as given in (6.6), and the relative shear $\tilde{q}_{\mu \nu}$, as given in (6.2), of the outgoing null vector to $\mathcal{B}_{t}$ (relative to their background values). Along with the hyperbolic angle $\Theta$ characterizing the initial velocity of $\mathcal{T}$ relative to $\mathcal{S}_{0}$, this data uniquely determines a harmonic spacetime metric (locally in time) by solving (5.1).

These boundary conditions have a hierarchical Sommerfeld form which is beneficial for numerical application. Although formally they can be applied to any metric-based evolution system, an unresolved issue is whether these boundary conditions, or some modification, are constraint preserving for hyperbolic reductions of the Einstein equations other than harmonic.

The geometrization of the boundary conditions for the gravitational IBVP has been presented in 4-dimensional form in which the data is prescribed in terms of the spacetime metric and its derivatives on $\mathcal{S}_{0}$ and $\mathcal{T}$. A question of further geometric interest is whether the data can also be presented in a disembodied 3-dimensional form, as is possible for the Cauchy problem by prescribing the initial data $h_{\mu \nu}$ and $k_{\mu \nu}$ intrinsic to the 3-manifold $\mathcal{S}_{0}$. Such Cauchy data then determines a spacetime metric solving Einstein's equations which is unique up to a diffeomorphism. If there is such a disembodied version of the boundary data for the IBVP in terms of the 3-manifolds $\mathcal{S}_{0}$ and $\mathcal{T}$, and their intersection $\mathcal{B}_{0}$, it does not appear that it can be as simple as in the pure Cauchy problem. In the treatment given here, the evolution vector $t^{\mu}$ plays key roles in both dealing with the diffeomorphism freedom and describing the boundary data in geometric form. The introduction of $t^{\mu}$ allows construction of a 4-dimensional background metric based upon the Cauchy data. A disembodied version would at the least require introducing $t^{\mu}$ on $\mathcal{T}$, along with the construction of a 3-dimensional background metric on $\mathcal{T}$. I leave it as an open question whether an equivalent 3 -dimensional version can be given.

\section{Acknowledgments}

This research was supported by NSF grant PH-0553597 to the University of Pittsburgh. Much of the work has been based upon ideas developed during previous collaborations with H-O. Kreiss, O. Reula and O. Sarbach. I have also profited from many discussions with H. Friedrich on the IBVP. Finally, my thanks to Jürgen, whose memory continues to be a guiding spirit.

[1] S. Kind and J. Ehlers, "Initial-boundary value problem for the spherically symmetric Einstein equations, for a perfect fluid", Class. Quantum Grav. 10, 2123 - 2136 (1993).

[2] S. Kind, J. Ehlers and B. G. Schmidt, "Relativistic stellar oscillations treated as an initial value problem", Class. Quantum Grav. 10, 2137 - 2152 (1993).

[3] H.O. Kreiss and J. Winicour, "Problems which are well-posed in a generalized sense with applications to the Einstein equations", Class. Quantum Grav.23, S405-S420 (2006).

[4] Y. Foures-Bruhat, "Theoreme d'existence pour certain systemes d'equations aux deriveés partielles nonlinaires", Acta Math 88, 141 (1952).

[5] J. M. Stewart, "The Cauchy problem and the initial boundary value problem in numerical relativity", Class. Quantum Grav. 15, 2865 (1998).

[6] H. Friedrich and G. Nagy, "The initial boundary value problem for Einstein's vacuum field equation", Commun. Math. Phys. 201, 619 (1999).

[7] M. C. Babiuc, H-O. Kreiss and J. Winicour, "Constraint-preserving Sommerfeld conditions for the harmonic Einstein equations", Phys. Rev. D 75, 044002 (2007).

[8] M. Shibata and T. Nakamura, "Evolution of three-dimensional gravitational waves: Harmonic slicing case", Phys. Rev. D 52, 5428 (1995).

[9] T. Baumgarte and S. L. Shapiro, "Numerical integration of Einstein's field equations", Phys. Rev. D 59, 024007 (1999).

[10] S.W. Hawking and G.F.R. Ellis, The Large Scale Structure of Space Time, Cambridge University Press, Cambridge (1973).

[11] H-O. Kreiss, O. Reula, O. Sarbach and J. Winicour "Boundary conditions for coupled quasilinear wave equations with application to isolated systems", Class. Quantum Grav. (to appear) (2009).

[12] "Well-posed initial-boundary value problem for the harmonic Einstein equations using energy estimates", H.-O. Kreiss, O. Reula, O. Sarbach, J. Winicour, Class. Quantum Grav. 24, 5973 (2007). 
[13] H.-O. Kreiss and J. Lorenz, Initial-Boundary Value Problems and the Navier-Stokes Equations, 1989, Reprint SIAM CLASSICS, 2004.

[14] Z. B. Etienne, J. A. Faber, Y. T. Liu, S. L. Shapiro, K. Taniguchi, T. W. Baumgarte, "Fully general relativistic simulations of black hole-neutron star mergers", Phys. Rev. D 77, 084002 (2008). 